\title{
EL PROCESO DE INVESTIGACIÓN: CONSTRUYENDO EL PROYECTO
}

\author{
THE RESEARCH PROCESS: \\ BUILDING THE PROJECT
}

Claudia Fabiola Ortega Barba

Doctora en Pedagogía, Facultad de Filosofía y Letras, Universidad Nacional Autónoma de México (UNAM). Maestra y licenciada en Ciencias de la Comunicación, Facultad de Ciencias Políticas y Sociales, UNAM. Profesora investigadora de tiempo completo de la Escuela de Pedagogía, Universidad Panamericana, campus Ciudad de México. Líneas de investigación: investigación educativa y mediaciones tecnológicas en procesos educativos para la innovación. Miembro del grupo de Investigación educación, instituciones e innovación, Escuela de Pedagogía, Universidad Panamericana, campus Ciudad de México. Miembro de la Red Mexicana de Investigadores de la Investigación Educativa (REDMIIE).

Correo electrónico: [cortega@up.edu.mx].

\section{RESUMEN}

Cuando se inicia una investigación, no importa bajo qué paradigma ${ }^{1}$ o enfoque se sustente, es necesario identificarla como un proceso sistemático de indagación o cuestionamiento de algo. Dicho proceso

1 Se entiende por paradigma a una tendencia científica que impacta en un momento determinado. Dicho concepto fue acuñado, a mediados del siglo XX, por Thomas Kuhn en su trabajo La estructura de las revoluciones cientificas. 
se configura de tres fases: planeación, realización y presentación; dichas fases no son lineales sino recursivas. Este trabajo se enfoca principalmente en la primera fase: el diseño del proyecto de investigación.

Palabras clave: investigación educativa; diseño del proyecto; formación de investigadores.

\section{ABSTRACT}

When a research is initiated, no matter under what paradigm or approach is to support, it is necessary to identify that the research is systematic process. This process is set in three phases: planning, implementation and presentation, these phases are recursive. This work focuses mainly on the first phase: the design of the research project.

Keywords: educational research; project design; training of researchers.

\section{DISEÑO DEL PROYECTO DE INVESTIGACIÓN}

En toda investigación, la primera fase es el diseño del proyecto de investigación. La investigación busca garantizar la viabilidad y funciona como guía. Específicamente en la planeación de la investigación se genera el diseño del proyecto, también llamado protocolo o anteproyecto (Arias, 2006), el cual se refiere a la primera etapa del proceso investigativo en donde se plasma qué se va a investigar, por qué se efectuará dicha investigación, para qué se investigará, cómo y con qué se realizará la investigación.

Cuando ya se ha avanzado en el proyecto, la segunda fase atiende a la investigación de campo o trabajo empírico, y finalmente la última etapa se relaciona con la presentación de la investigación en un informe o reporte. 
Para comenzar una investigación es indispensable traducir las preocupaciones que se tienen acerca de la realidad y sobre las cuales se pretende realizar una indagatoria científica como una problemática.

\section{PLANTEAMIENTO DEL PROBLEMA}

La problemática expresa una situación reconocida, puesta bajo estudio por el investigador. Responde a la pregunta: ¿cuál es el caso que llama la atención de donde surge el tema a investigar? Para formalizar dicha problemática es necesario empezar, en paralelo, a hacer uso de la investigación documental; lo anterior permite confrontar la realidad del investigador con otras realidades ya estudiadas, e ir integrando el marco de referencia de la investigación.

Para desarrollar la problemática, habrá que identificar el problema. Éste lo entenderemos como la relación de variables ${ }^{2}$ o categorías $^{3}$ pensadas por el investigador: del problema se desprende el tema de investigación y éste se cristaliza, en un primer momento, como el título de la misma ${ }^{4}$.

\section{JUSTIFICACIÓN}

A la par de la identificación del problema, surge otra pregunta a responder con la investigación y que corresponde a la justificación: ¿por qué me interesa investigar esto? La justificación responde al por qué, que puede ser: teórico, metodológico o práctico (Martínez, 2004, p. 103). Si es teórico debe responder a las siguientes preguntas: ¿quiere ampliar una teoría?, ¿quiere contrastar la teoría con la realidad?, ¿quiere refutar

2 El concepto de variable hace referencia a investigaciones de corte cuantitativo y se entiende como una característica observable que se puede medir.

3 El concepto de categoría es propio de investigaciones con enfoque cualitativo. Las categorías son los conceptos en la investigación que se emplearán para delimitar el tema.

4 El título de la investigación puede irse modificando conforme el proceso avanza y de acuerdo a las necesidades propias del objeto de estudio. 
la validez de una teoría con la realidad?, ¿quiere reafirmar la validez de una teoría con la realidad?, ¿quiere presentar un complemento teórico de aquél en el cual fundamenta su investigación?

Si es metodológico deberá responder preguntas como: ¿es un instrumento o un material que podrá ser empleado?, ¿permite explicar la validez de un instrumento o un material?

Si es práctico puede responder a: ¿el resultado de la investigación tiene una aplicación concreta?, ¿el resultado de la investigación ayudará a mejorar los procedimientos de una organización?, ¿el resultado de la investigación ayudará a resolver problemas en una organización?, ¿el resultado de la investigación mejorará alguna situación actual?

Una forma de justificar un estudio es a partir del número de involucrados en la realidad a estudiar, por ello se sugiere consultar documentos con cifras, como por ejemplo, los generados por el Instituto Nacional de Estadística, Geografía e Informática (INEGI), o algunas investigaciones anteriores.

\section{MARCO DE REFERENCIA}

El siguiente paso a la identificación del problema es la formalización del mismo mediante el trabajo documental que permitirá construir un marco de referencia (Lafrancesco, 2003, p. 57); éste es clave en cualquier investigación. Para llevarlo a cabo se recurre, en primera instancia, a los pasos de la investigación documental: contar con un tema; acudir a las instituciones pertinentes (físicas ${ }^{5}$ o digitales ${ }^{6}$ ); consultar las fuentes;

5 Nos referimos a bibliotecas, hemerotecas, videotecas, fonotecas, iconotecas, mapotecas, por citar algunas.

6 Nos referimos a bases de datos con fuentes hemerográficas y bibliográficas de corte científico y académico, como EBSCO, ScienceDirect, Dialnet, Jstore, Springer, Scielo y la Red de Revistas Científicas de América Latina y el Caribe, España y Portugal (REDALyC), por citar algunas. 
realizar esquemas y notas (fichas) y redactar ${ }^{7}$. Con el propósito de seleccionar las fuentes que servirán para la elaboración del marco de referencia, conviene partir de las siguientes preguntas: ¿cómo se relaciona las referencias con mi problema de investigación?, ¿las fuentes consultadas me ayudan a desarrollar mi investigación?, ¿desde qué perspectiva abordan el tema?

Elaborar el marco de referencia ayuda a plantear adecuadamente el problema, pues integra el estado del $\operatorname{arte}^{8}$, la teoría ${ }^{9}$ (marco teórico), los conceptos $^{10}$ (marco conceptual) y el contexto (marco situacional).

El marco de referencia implica analizar y exponer la teoría, buscar los conceptos y antecedentes válidos en otras investigaciones para encuadrar el estudio; es decir, sustentarlo teórica ${ }^{11} \mathrm{y}$ conceptualmente.

En la ciencia, la experiencia vale siempre que esté iluminada por la teoría, es decir, la unión entre el trabajo de campo o proceso empírico y la estructura formal de la ciencia adquieren significado al estar estrechamente vinculados.

Las funciones del marco de referencia son: prevenir errores; orientar sobre el estudio; ampliar el horizonte de estudio; inspirar nuevas líneas de investigación, y proveer de un marco que sustente y explique

7 Al redactar es importante considerar el uso adecuado del aparato crítico, el cual está compuesto por citas, referencias y notas. En cuanto al sistema de referencias, se sugiere elegir uno antes de iniciar con la redacción del documento. Existen diversos sistemas de referencia como: American Psychological Association (APA), Modern Language Association (MLA), Turabian, Vancouver, International Organization for Standardization (ISO), Harvard y Chicago, entre otros.

8 También llamado estado de la cuestión o revisión de la literatura, es la revisión de trabajos similares. La recopilación de información sobre otras investigaciones relacionadas con el tema a estudiar se le llama estado del arte o estado de la cuestión.

9 Se entiende por teoría una explicación amplia de la realidad. Ejemplos de teoría: la teoría conductista, la teoría de sistemas... Es importante no confundir el término teoría con el término teórico, que muchas veces se utiliza para contraponer al término práctico.

10 Se entiende por concepto una idea abstracta o mental con el significado de las variables (si es un trabajo cuantitativo) o categorías (si es una investigación cualitativa) del trabajo.

11 Cabe aclarar que sustentar teóricamente el estudio no es crear teoría. 
la problemática a estudiar. Específicamente el marco de referencia contesta la pregunta ¿qué voy a investigar? Aunado a éste se encuentra la pregunta de investigación y/o la hipótesis.

\section{PREGUNTA DE INVESTIGACIÓN}

En trabajos con un enfoque cualitativo ${ }^{12}$, la pregunta de investigación es la guía de lo que se va a investigar; mientras que es la hipótesis la que guía los trabajos con enfoque cuantitativo.

En la pregunta de investigación se requiere tomar en cuenta las categorías, las unidades de observación ${ }^{13}$ y, de ser necesario, los límites espaciales y temporales. Durante el desarrollo de la investigación puede cambiarse la pregunta original si se ha modificado el tema. Muchas veces, cuando se pregunta qué interesa investigar, lo primero que viene a la mente es una pregunta, misma que puede transformarse en el título del trabajo o viceversa, como se muestra en el siguiente ejemplo:

Tema: La relación entre el grado de escolaridad y la participación en organizaciones políticas de los jóvenes universitarios

Se desprende del tema $y$ del problema

\section{Pregunta de investigación:¿Cuál es la relación entre el grado de escolaridad y la participación en organizaciones políticas de los jóvenes universitarios?}

12 El objetivo del enfoque cualitativo es profundizar en una situación problemática (Galeano, 2004, p. 21).

13 Se refiere a los actores de la problemática a estudiar. 
Para revisar si la pregunta está planteada correctamente será necesario verificar que no se formulen respuestas cerradas como «síl» o «no», pues recordemos que éstas sirven para guiar la investigación; inclusive existen autores que las entienden como el planteamiento del problema en sí mismas.

Ahora bien, la pregunta de investigación es el componente que guía los trabajos cualitativos (Strauss y Corbin, 2002, p. 40). Sin embargo, puede utilizarse en trabajos cuantitativos; de hacerlo, en lugar de tener una respuesta abierta, tendrá una respuesta cerrada, como por ejemplo: ¿existe correlación entre el grado de escolaridad y la participación en organizaciones políticas de los jóvenes universitarios?, la respuesta a dicha pregunta se complementa con la hipótesis.

Como ya se ha mencionado, los trabajos cualitativos se guían por la pregunta de investigación, mientras que los cuantitativos tienen como base las hipótesis. En este apartado se busca explicar qué es una hipótesis, cuáles son los tipos de hipótesis y cómo se elabora cada tipo. Lo anterior ayudará al estudiante a redactar la hipótesis, en caso de necesitarla ${ }^{14}$.

\section{HIPÓTESIS}

La hipótesis es una explicación tentativa a un fenómeno de investigación sujeta a comprobación empírica, o sea, una suposición verosímil, luego comprobable o rebatible: «La hipótesis consiste en suponer conocida la verdad o explicación que se busca» (Cervo y Bervian, 1998, pp. 23-24). Es muy importante tener en cuenta que las hipótesis se utilizan en trabajos donde se busca la comprobación de lo que se está planteando, trabajos con enfoque cuantitativo.

La hipótesis se formula a manera de proposición ${ }^{15}$ o enunciado contundente y debe estar compuesto de variables. La variable es una

\footnotetext{
14 Cabe aclarar que no todos los trabajos de investigación requieren hipótesis. Ésta se emplean, específicamente, para trabajos cuantitativos.

15 Las proposiciones son frases compuestas de conceptos y palabras de enlace.
} 
característica, atributo o propiedad observable la cual refleja o expresa algún concepto, y admite diferentes valores, pues tiende a cambiar y además debe medirse.

Al formular una hipótesis es indispensable definir las variables o los términos incluidos en ésta. Las variables deben presentar dos definiciones: una conceptual, consecutiva o constitutiva, y otra operacional. La primera se refiere a la conceptualización de la variable. La segunda traduce los conceptos a hechos observables y presenta la forma como se medirá.

La variable posee como características: referirse a una situación real; ser concreta; ser simple, con una relación entre variables-verosímil, y ser medible.

Ejemplo de variable:

\begin{tabular}{|l|l|l|}
\hline Variable & Definición conceptual & Definición operacional \\
\hline Memoria: & $\begin{array}{l}\text { Es la función cerebral } \\
\text { resultado de conexio- } \\
\text { nes sinápticas entre } \\
\text { neuronas, mediante la } \\
\text { que el ser humano pue- } \\
\text { de retener experiencias numérica. } \\
\text { pasadas. }\end{array}$ & Naceso. \\
\hline
\end{tabular}

Existen distintos tipos de hipótesis con las cuales se trabaja en una investigación. Éstas son: de trabajo, nulas y alternativas. Dentro de las hipótesis de trabajo podemos encontrar las hipótesis: 1) descriptivas, que involucran una sola variable; 2) correlacionales, que involucran dos o más variables en forma de asociación, y 3) de causalidad, que relacionan dos o más variables en términos de dependencia (Rojas, 2006, pp. 150-154). 
Hipótesis de investigación o de trabajo. Son las más comunes y se suelen simbolizar como Hi o H1, H2, H3..., y se suelen clasificar de acuerdo al número y relación entre variables en:

- Descriptivas, son afirmaciones en donde sólo plantea cómo se manifiestan las variables en un contexto. Ejemplo: Los niños de la calle son analfabetos.

- Correlacionales, especifican las relaciones, vinculaciones o asociaciones entre $\operatorname{dos}^{16} \mathrm{o}$ más variables ${ }^{17}$, y el cómo de las mismas. En este tipo de hipótesis, el orden de colocación de las variables no importa. Éstas pueden redactarse: a mayor $X$, mayor $Y$; a mayor $Y$, mayor $X$; a mayor $X$, menor $Y$; a mayor $Y$, mayor $X$. Ejemplo: A mayor cultura fiscal, mayor recaudación de impuestos. A mayor recaudación de impuestos, mayor cultura fiscal.

- De relaciones de causalidad, en éstas sí importa el orden de las variables, pues una es causa, y otra, efecto; a la primera se le conoce como «variable independiente» y a la segunda como «variable dependiente». Este tipo de hipótesis afirma relaciones entre dos o más variables y cómo se originan dichas relaciones.

Hipótesis nulas. Constituyen proposiciones opuestas o complementarias a las hipótesis de trabajo. Debido a que este tipo de hipótesis resulta de la contrapartida de la hipótesis de investigación, existen tantas clases de hipótesis nulas como de investigación. Se simbolizan como: $\mathrm{Ho}$

Hipótesis alternativas. Son hipótesis de investigación adicionales a la hipótesis original, es decir, son otras posibilidades distintas a las hipótesis de trabajo o a la hipótesis nula. Ofrecen otra descripción o explicación distinta de la proporcionada por las anteriores. Éstas se simbolizan con $\mathrm{Ha}$ y sólo pueden formularse cuando efectivamente existen otras posibilidades, de no ser así, entonces se omiten. Ejemplo:

16 Cuando se correlacionan dos variables se conoce como correlación bivariada.

17 Cuando se correlacionan más de dos variables se conoce como correlación múltiple. 
Hi: Los niños de la calle de la Ciudad de México son analfabetos.

Ho: Los niños de la calle de la Ciudad de México no son analfabetos.

Ha: Los niños de la calle de la Ciudad de México están desnutridos.

Finalmente es importante recalcar que cada investigación requerirá diversos tipos de hipótesis de trabajo según el carácter de la misma.

\section{OBJETIVOS}

Aunado a la pregunta de investigación o hipótesis de trabajo, dependiendo el carácter de la investigación, cualitativa o cuantitativa respectivamente, se presenta el objetivo, éste indica lo que se pretende alcanzar con la investigación y se redacta iniciando con un verbo en infinitivo. Para formular el objetivo se requiere: tener el tema delimitado, un marco de referencia y la pregunta de investigación o hipótesis.

Los objetivos responden a las preguntas: ¿qué quiero hacer en la investigación?, ¿qué es lo que busco?, ¿a dónde quiero llegar?

Con el propósito de facilitar la redacción de los objetivos en un trabajo de investigación, a continuación se presenta una lista de verbos, los cuales pueden servir como referencia para dicha elaboración:

\begin{tabular}{|l|l|l|l|}
\hline Analizar & Establecer & Producir \\
\hline Compilar & Especificar & Programar \\
\hline Completar & Estandarizar & Propiciar \\
\hline Comprobar & Evaluar & Proporcionar \\
\hline Conocer & Examinar & Proveer \\
\hline Consolidar & Formular & Redactar \\
\hline Consultar & Hacer & Someter \\
\hline Contestar & Identificar & Verificar \\
\hline
\end{tabular}


Para cubrir el objetivo general se requiere plantear algunos objetivos específicos que permitirán cubrir el general (Tamayo, 2004, p. 140).

Ejemplo.-

Tema: Modelo de uso de tecnologías informáticas para docentes universitarios.

Objetivo general: Diseñar un modelo de uso de tecnologías informáticas para docentes universitarios.

Objetivos particulares: Enumerar las tecnologías informáticas al servicio de la enseñanza. Conocer los usos que los docentes universitarios hacen de la tecnología.

\section{METODOLOGÍA}

Cuando ya se tiene la problemática identificada, el problema delimitado, el marco de referencia, la pregunta de investigación y/o la hipótesis y los objetivos, el siguiente paso en el proceso es el cómo responder a la pregunta: ¿cómo se realizará la investigación? Ello se trabaja mediante la metodología en donde se identifican los principios generales que han de guiar el trabajo. La metodología estudia, define, construye y / o valida los métodos y las técnicas a utilizar en la investigación. La metodología, entendida como el estudio de los métodos, da sentido, pertinencia, rigor y viabilidad a una investigación.

La metodología se compone principalmente del conjunto de métodos y técnicas a utilizar. El método es el modo de hacer, obrar o proceder; es decir, el camino a seguir para cubrir los objetivos planteados. Éste incluye un momento instrumental al que antecede todo un trabajo de reflexión, por lo que el método se constituye por un lado de los procedimientos, técnicas e instrumentos, y por el otro de los fundamentos teóricos y criterios lógicos.

Ahora bien, las técnicas son aquellas herramientas específicas utilizadas para recopilar y analizar la información. 
Cuando se trabaja sobre la metodología se debe contemplar el tipo de investigación a realizar. A continuación se presentan sólo algunas clasificaciones.

Según el propósito: Básica, fundamental o pura o aplicada, activa o dinámica.

Según el enfoque: cuantitativa (experimental, cuasi experimental o ex post facto); cualitativa (etnografía, investigación acción, historia de vida, teoría fundamentada), o mixta.

Resuelto todo lo anterior, el siguiente paso es la entrada al campo para la recolección de la información. En trabajos cuantitativos por lo regular se recurre a técnicas como los test, las listas de control o las encuestas; mientras que para los trabajos cualitativos se utiliza la entrevista, la observación y los documentos.

\section{A MANERA DE CIERRE}

Cuando ya se cuenta con la información empírica o de campo, lo siguiente es el análisis de los datos; para trabajos cuantitativos se emplea la estadística descriptiva e inferencial ${ }^{18}$, mientras que para los trabajos cualitativos se trabaja con el análisis interpretativo.

A la par que se realiza la investigación se trabaja la presentación del informe que mostrará, en la primera parte, el marco de referencia; en la segunda, la metodología, y en la tercera parte, el análisis de la información y las conclusiones.

Con todo lo expuesto se ha realizado un recorrido sintético del proceso de investigación, desde el origen de la problemática, hasta la presentación del documento de investigación.

18 Para este tema puede revisarse el libro de González, M.C. y Medina L. (2015). Alicia en el país de las estadísticas con $R$ y Excel, México: FES Acatlán-UNAM. 


\section{FUENTES DE CONSULTA}

Arias, F. (2006). El proyecto de investigación: Guía para su elaboración, $5^{\text {a }}$ ed. Caracas: Episteme.

Cervo, A. y Bervian, P. (1998). Metodología científica. México: McGraw Hill.

Galeano, M. (2004). Diseño de proyectos en la investigación cualitativa. Medellín: Fondo Editorial Universidad EAFIT.

González, M.C. y Medina L. (2015), Alicia en el país de las estadísticas con $R$ y Excel. México: FES Acatlán-UNAM.

Kuhn, T. (1986). La estructura de las revoluciones cientificas, $7^{a}$. reimpr. México: Fondo de Cultura Económica.

Lafrancesco, G. (2003). La investigación en educación y Pedagogía. Fundamentos y técnicas. Colombia: Escuela Transformadora Magisterio.

Martínez, V. (2004). Fundamentos teóricos para el proceso de diseño de un protocolo de investigación, $1^{\mathrm{a}}$. reimpr. de la $2^{\mathrm{a}}$. ed. México: Plaza y Valdés.

Rojas, R. (2006). Guía para realizar investigaciones sociales, 33ava. ed. México: Plaza y Valdez.

Strauss, A. y Corbin, J. (2002). Bases de la investigación cualitativa. Técnicas y procedimientos para desarrollar la teoría fundamentada, $1^{\text {a }}$. ed. en español. Colombia: Universidad de Antioquia.

Tamayo, M. (2004). El proceso de la investigación científica, $4^{\mathrm{a}}$. ed. México: Limusa. 